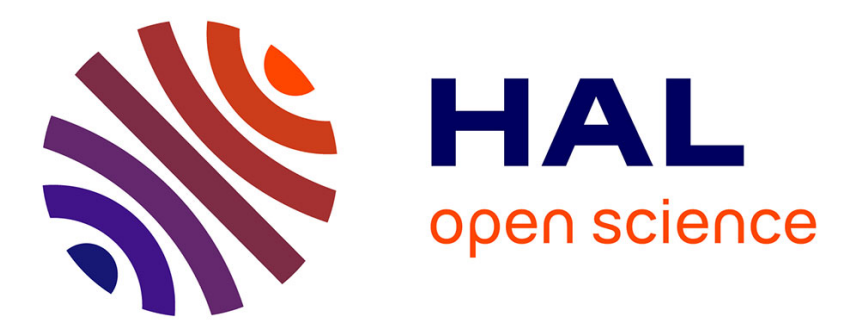

\title{
Evolution de la texture et de la microstructure d'un aluminiure de titane biphase $\gamma / \alpha 2$ en fonction des traitements thermiques
}

\author{
M.-L. Dupont, T. Baudin, R. Penelle, S. Naka, M. Thomas
}

\section{To cite this version:}

M.-L. Dupont, T. Baudin, R. Penelle, S. Naka, M. Thomas. Evolution de la texture et de la microstructure d'un aluminiure de titane biphase $\gamma / \alpha 2$ en fonction des traitements thermiques. Journal de Physique IV Proceedings, 1996, 06 (C2), pp.C2-235-C2-240. 10.1051/jp4:1996233 . jpa-00254211

\section{HAL Id: jpa-00254211 https://hal.science/jpa-00254211}

Submitted on 1 Jan 1996

HAL is a multi-disciplinary open access archive for the deposit and dissemination of scientific research documents, whether they are published or not. The documents may come from teaching and research institutions in France or abroad, or from public or private research centers.
L'archive ouverte pluridisciplinaire HAL, est destinée au dépôt et à la diffusion de documents scientifiques de niveau recherche, publiés ou non, émanant des établissements d'enseignement et de recherche français ou étrangers, des laboratoires publics ou privés. 


\title{
Evolution de la texture et de la microstructure d'un aluminiure de titane biphase $\gamma / \alpha_{2}$ en fonction des traitements thermiques
}

\author{
M.-L. Dupont, T. Baudin, R. Penelle, S. Naka* et M. Thomas* \\ Laboratoire de Métallurgie Structurale, U.R.A. 1107 du C.N.R.S., Université Paris-Sud, Bât. 413, \\ 91405 Orsay Cedex, France \\ * Office National d'Etudes et de Recherches Aérospatiales, 29 Av. de la Division Leclerc, 92322 Châtillon \\ Cedex, France
}

\begin{abstract}
Titanium aluminides are good candidates for aeronautical applications, but their low ductility penalizes these alloys. The present work deals with the texture characterization that influences mechanical properties. The texture is a $\{111\}<u v w>$ fiber texture, the sharpness of which increases with the temperature of heat treatments. $\gamma$ monolithic grains have also the same texture.
\end{abstract}

\section{INTRODUCTION}

Les aluminiures de titane sont des composés intermétalliques faisant actuellement l'objet de nombreuses recherches. En effet, leur faible densité alliée à de bonnes caractéristiques mécaniques à haute température et une résistance élevée à l'oxydation, en font des alliages susceptibles d'être utilisés en aéronautique. Mais leur principal défaut est leur faible ductilité à basse température jusqu'à environ $500^{\circ} \mathrm{C},[1]$.

Les alliages qui nous intéressent sont des alliages biphasés $\gamma / \alpha_{2}$ où $\gamma$ est lintermétallique TiAl de structure quadratique et $\alpha_{2}$ l'intermétallique $\mathrm{Ti}_{3} \mathrm{Al}$ de structure hexagonale. Yamaguchi et Inui [2] ont montré sur un pseudo-monocristal que la ductilité peut varier de façon très importante suivant l'angle entre l'axe de sollicitation et le plan des lamelles $\left(\gamma / \alpha_{2}\right)$. C'est pourquoi nous nous intéressons à la texture morphologique et cristallographique de cette catégorie d'alliages. L'étude porte sur l'évolution de la texture et de la microstructure en fonction des différents traitements thermiques. Ces traitements ont pour but d'essayer de modifier, la texture afin de pouvoir comprendre par la suite son influence sur les propriétés mécaniques.

L'alliage qui fait l'objet de la présente étude est un alliage de General Electric de composition atomique $48 \% \mathrm{Ti}-48 \% \mathrm{Al}-2 \% \mathrm{Cr}-2 \% \mathrm{Nb}$. La texture des échantillons est déterminée par deux techniques différentes et complémentaires : la diffraction des neutrons d'une part et la diffraction des électrons rétrodiffusés (Electron BackScattered Diffraction, EBSD) d'autre part. La diffraction des neutrons donne la texture globale tandis que l'EBSD donne la texture locale et permet de différencier la texture de chacune des composantes de la microstructure.

\section{TECHNIQUES ET RESULTATS EXPERIMENTAUX}

Les échantillons étudiés sont des orthocylindres de $15 \mathrm{~mm}$ de diamètre prélevés, dans la zone de solidification colonnaire, perpendiculairement à l'axe du lingot brut de coulée ayant subi un compactage isostatique à chaud $\left(1190\right.$ bars, $\left.1180^{\circ} \mathrm{C}\right)$. Les traitements thermiques sont réalisés sous atmosphère d'argon dans différents domaines du diagramme de phases (figure 1 et tableau 1). 


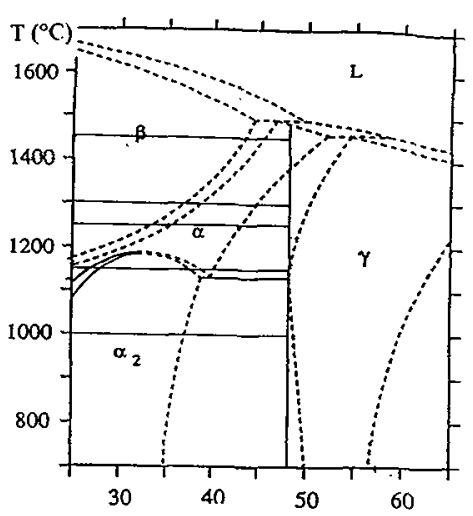

$\mathrm{Ti} \%$ atomique $\mathrm{Al}$

\begin{tabular}{|c|c|c|}
\hline Température & Temps & Refroid \\
\hline $1000^{\circ} \mathrm{C}$ & $24 \mathrm{~h}$ & four \\
\hline $1150^{\circ} \mathrm{C}$ & $4 \mathrm{~h}$ & four \\
\hline $1150^{\circ} \mathrm{C}$ & $24 \mathrm{~h}$ & four \\
\hline $1250^{\circ} \mathrm{C}$ & $4 \mathrm{~h}$ & four \\
\hline $1250^{\circ} \mathrm{C}$ & $24 \mathrm{~h}$ & four \\
\hline $1300^{\circ} \mathrm{C}$ & $4 \mathrm{~h}$ & four \\
\hline $1300^{\circ} \mathrm{C}$ & $24 \mathrm{~h}$ & four \\
\hline $1450^{\circ} \mathrm{C}$ & $1 \mathrm{~h}$ & four \\
\hline $1450^{\circ} \mathrm{C}$ & $1 \mathrm{~h}$ & trempe huile \\
\hline
\end{tabular}

tableau $1:$ traitements thermiques (refroidissement four coupé $\approx 0.05^{\circ} \mathrm{C} / \mathrm{s}$ )

figure 1: diagramme de phases $\mathrm{Ti}-\mathrm{Al},[3]$

\subsection{Microstructure}

La microstructure est étudiée à la fois par microscopie optique en contrate de Nomarski et par microscopie électronique à balayage (MEB) sur des échantillons polis électrolytiquement $15 \mathrm{~min}$ à $-50^{\circ} \mathrm{C}$ sous $24 \mathrm{~V}$ dans la solution proposée par Blackburn et Williams, [4]. La microstructure type des échantillons à l'état brut de livraison est montrée sur la figure 2. Elle se compose de grains "monolithiques" $\gamma$ très souvent maclés et de tailles variées : de $5 \mu \mathrm{m}$ environ à 50 voire $100 \mu \mathrm{m}$ et de gros grains lamellaires de l'ordre de $500 \mu \mathrm{m}$, avec alternance de lamelles $\gamma$ et $\alpha_{2}$. Ainsi que l'ont déjà observé Denquin et Naka $[5,6]$, plusieurs lamelles $\gamma$ sont souvent accolées.

Cette microstructure évolue peu avec les traitements thermiques (excepté pour $1450^{\circ} \mathrm{C}$, domaine monophasé $\alpha$ ) dans la mesure où l'on n'observe pas d'augmentation de la taille des grains lamellaires et que l'on observe toujours des grains monolithiques pour tous les traitements en domaine biphasé : l'état de départ.

- à $1000^{\circ} \mathrm{C}$ (domaine $\alpha_{2}+\gamma$ ), on n'observe pas d'évolution de la microstructure comparativement à

- à $1150^{\circ} \mathrm{C}$ (domaine $\alpha+\gamma$, juste au-dessus du palier eutectoïde), on trouve une quantité plus importante de grains monolithiques (à $1000^{\circ} \mathrm{C}$, la diffusion serait trop lente pour qu'il y ait croissance de ces derniers). La croissance en épaisseur des lamelles étant limitée par la présence de joints de macles et de lamelles $\alpha$, le traitement thermique à cette température $\left(1150^{\circ} \mathrm{C}\right)$ a surtout pour effet d'augmenter la fraction surfacique des grains monolithiques, (figure 3).

- au cours des traitements thermiques à $1250^{\circ} \mathrm{C}$ ou $1300^{\circ} \mathrm{C}$, la proportion de phase $\alpha$ devenant plus importante, cette phase précipite dans les grains monolithiques (figure 4). Dans un même grain, la phase $\alpha$ apparaît sous forme d'aiguilles orientées le plus souvent suivant 3 directions privilégiées, correspondant probablement aux relations d'orientation possibles entre les phases $\alpha$ et $\gamma\left(3\right.$ directions $\langle 110\rangle_{\gamma} / /\langle 1120\rangle_{\alpha 2}$ dans un plan $\left.\{111\}_{\gamma} / /(0001)_{\alpha z}\right)$.

- après traitement dans le domaine monophasé $\alpha\left(1450^{\circ} \mathrm{C}\right)$, quelle que soit la vitesse de refroidissement adoptée, les échantillons présentent une microstructure vraiment différente : les grains 
monolithiques disparaissent et donc seuls les grains lamellaires se reforment au refroidissement et sont de taille plus importante (de l'ordre du $\mathrm{mm}$ ).

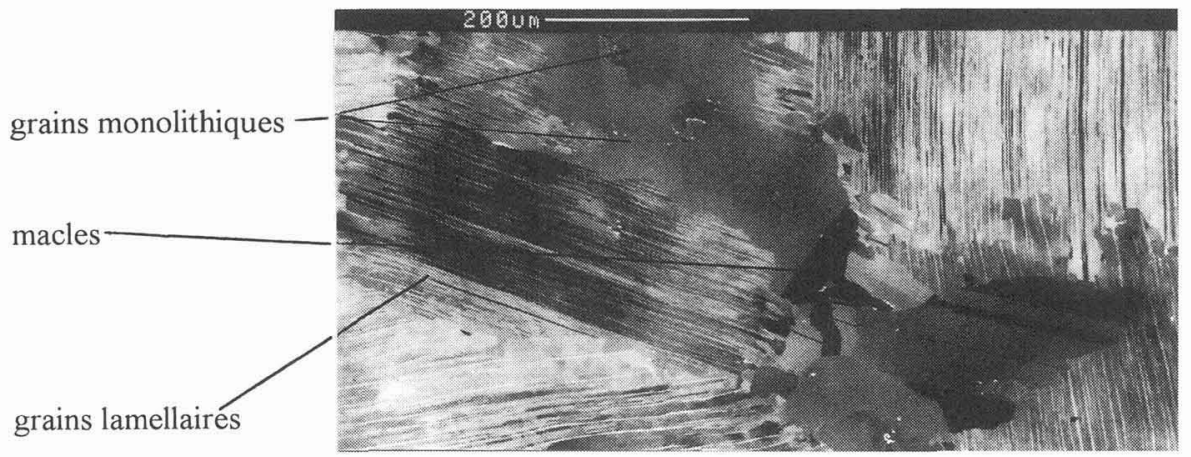

figure 2 : microstructure type de l'alliage $\mathrm{Ti}-48 \mathrm{Al}-2 \mathrm{Cr}-2 \mathrm{Nb}$

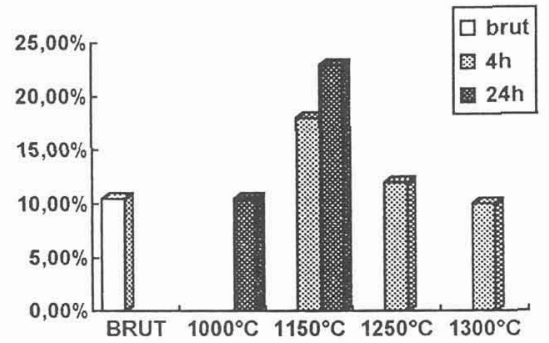

figure 3 : evolution du pourcentage surfacique de $\gamma$ monolithique en fonction de la température et de la durée de traitement

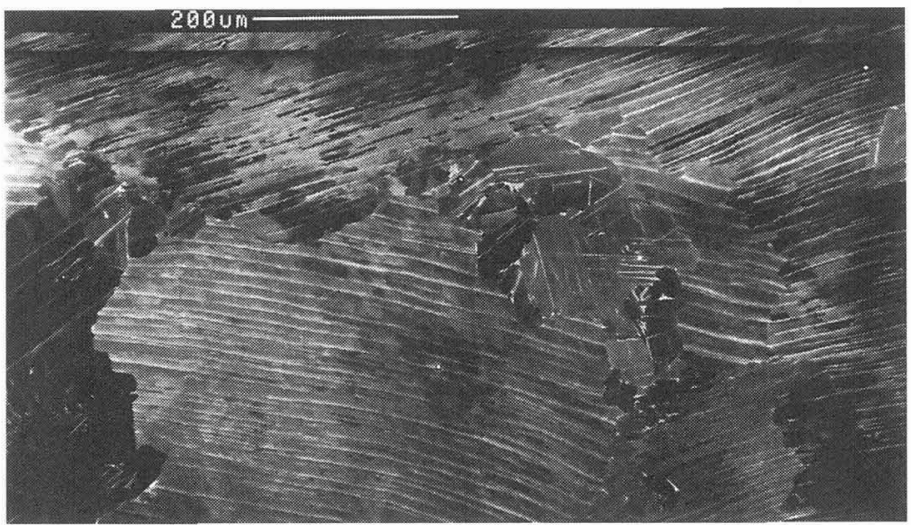

figure 4 : microstructure après un traitement de $4 \mathrm{~h}$ à $1250^{\circ} \mathrm{C}$ 


\subsection{Texture}

\subsubsection{Diffraction des neutrons}

La taille des grains lamellaires étant importante (au moins $500 \mu \mathrm{m}$ ), l'utilisation de rayons $\mathrm{X}$ est impossible d'un point de vue statistique (diffraction en surface), c'est pourquoi nous faisons appel à la diffraction des neutrons (diffraction en volume).

Six figures de pôles sont nécessaires pour chaque échantillon afin de pouvoir ensuite calculer la fonction de distribution des orientations cristallines (FDOC) par la méthode harmonique avec un ordre de développement $L=18$. Ces six figures sont $\{001\},\{110\},\{111\},\{201\},\{112\},\{221\}$, [7]. Comme la proportion de la phase $\alpha_{2}$ est très faible $(\approx 5 \%)$, elle ne peut pas être détectée, il n'y a donc pas de risque de convolution entre les pics de $\gamma$ et ceux de $\alpha_{2}$.

Nous avons pu ainsi caractériser la texture et montrer la présence d'une texture de fibre de type $\{111\}<u v w>$ dont l'acuité tend à augmenter avec la température et la durée de traitement (figure 5).

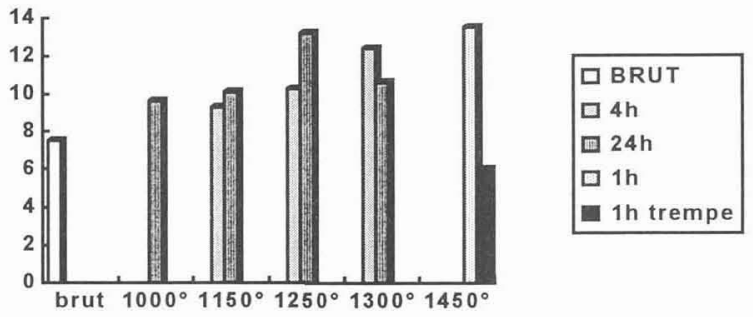

figure 5 : évolution de l'intensité maximum des FDOC

\subsubsection{Diffraction des électrons rétrodiffusés}

La préparation des échantillons est la même que celle adoptée pour l'observation des microstructures au MEB. L'EBSD a permis de séparer la texture des grains monolithiques de celle des grains lamellaires, (figure 6), [8], Par contre, il n'a pas été possible de déterminer la texture de la phase $\alpha_{2}$, les lamelles étant trop fines $(<0,5 \mu \mathrm{m})$ vis à vis de la résolution spatiale de la technique $(0,5 \mu \mathrm{m})$.

L'étude a permis de montrer que les grains monolithiques sont texturés et que leur texture est la même que celle des grains lamellaires, globalement équivalente à la texture mesurée aux neutrons. Ceci est observé quel que soit le traitement, excepté pour $1450^{\circ} \mathrm{C}$.

Si une texture des grains lamellaires est attendue (formation liée aux conditions d'élaboration), il convient, pour expliquer la texture des grains monolithiques, de faire appel aux hypothèses émises concernant leur origine $[3,9]$
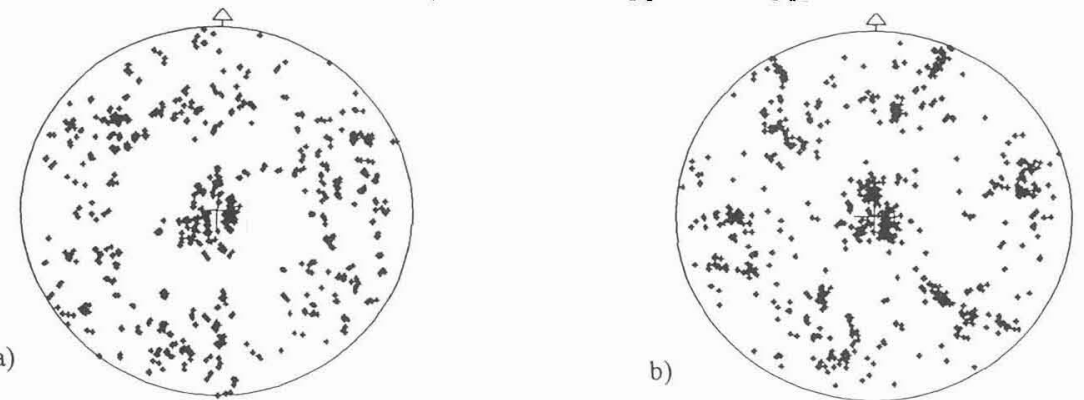

figure 6 : figures de pôles $\{111\}$ obtenues par EBSD sur un échantillon traité $24 \mathrm{~h}$ à $1000^{\circ} \mathrm{C}$, a) grains lamellaires, b) grains monolithiques 


\section{FORMATION DES GRAINS MONOLITHIQUES - DISCUSSION}

D'après le diagramme d'équilibre, et si l'on ne tient pas compte du passage dans le domaine $\beta+\mathrm{L}$ (ce qui ne gène pas le raisonnement), au cours de la solidification du lingot, il y aurait tout d'abord germination puis croissance de dendrites $\alpha$ avec enrichissement progressif du liquide en Al. Lors du passage dans le domaine $\alpha+\gamma$, du $\gamma$ enrichi en $\mathrm{Al}$ précipiterait aux joints des grains $\alpha$ sous forme de grains monolithiques, les grains lamellaires se formant ultérieurement.

Nous avons constaté que les grains monolithiques sont généralement situés d'un seul et même côté du joint et qu'ils ont la même orientation que les lamelles auxquelles ils sont accolés.

Il existe une relation d'orientation entre $\alpha_{2}$ et $\gamma$ :

$$
<1120>_{\alpha 2} / /<110>_{\gamma} \text { et }\{0001\}_{\alpha 2} / /\{111\}_{\gamma}
$$

Comme les grains $\alpha$ colonnaires (les échantillons sont prélevés dans la zone basaltique du lingot) se solidifient selon la direction [0001] (direction de croissance privilégiée pour les alliages de structure hexagonale) perpendiculaire à l'axe du lingot donc parallèle à l'axe de l'échantillon, les plans $\{111\}$ de $\gamma$ sont donc, compte-tenu de la relation d'orientation, perpendiculaires à l'axe [0001]. La texture des grains lamellaires est donc bien du type $\{111\}$.

L'orientation des grains monolithiques étant la même que celle des grains lamellaires, il s'en suit donc que ces grains sont également en relation d'orientation avec les grains $\alpha$ d'origine. Ceci explique qu'ils ont des orientations privilégiées et que leur texture est la même que celle des grains lamellaires.

L'hypothèse de l'enrichissement des grains monolithiques en $\mathrm{Al}$ expliquant leur formation reste à vérifier par microanalyse. Elle est cependant étayée par le fait qu'un traitement à $1450^{\circ} \mathrm{C}$ dans le domaine monophasé $\alpha$ homogénéise la composition et qu'alors, seuls les grains lamellaires soient observés.

\section{CONCLUSIONS - PERSPECTIVES}

La texture de l'alliage General Electric, de composition $\mathrm{Ti}-48 \% \mathrm{Al}-2 \% \mathrm{Cr}-2 \% \mathrm{Nb}$, brut de solidification ayant subi un compactage isostatique à chaud, est une texture de fibre de type $\{111\}<u v w>$ dont l'acuité augmente avec la température et/ou la durée des traitements ultérieurs. Les grains monolithiques dans cet alliage sont également texturés et de même texture que les grains lamellaires. Nous pouvons dire qu'il est impossible de modifier la texture par simple traitement thermique. Une étude sur des échantillons déformés à chaud est en cours actuellement.

Une étude plus fine (par EBSD) devrait permettre de comprendre l'origine de l'augmentation de l'acuité de la texture globale.

\section{Références}

[1] Y.W. Kim, JOM July (1994).30-39

[2] M. Yamaguchi and $\mathrm{H}$. Inui, " Deformation behavior of $\mathrm{TiAl}$ compounds with the $\mathrm{TiAl} / \mathrm{Ti}_{3} \mathrm{Al}$ lamellar microstructure", Ordered Intermetallics - Physical Metallurgy and Mechanical Behaviour, Irsee 23-28 June 1991, C.T. Liu and al.eds.(Kluwer Academic Publishers, Dordrecht, 1992) pp.217-235

[3] C. McCullough, J.J. Valencia, C.G. Levi and R. Mehrabian, Acta metall. 375 (1989) 1321-1336

[4] M.J. Blackburn and J.C. Williams, Trans AIME 239 (1967) 287-288 
[5] A. Denquin, thèse de doctorat, université de Lille, 1994

[6] A. Denquin, S. Naka and T. Khan, 'Transformation kinetics in $\gamma+\alpha_{2}$ TiAl-based alloys", Titanium '92Science and Technology, F.H. Froes and I. Caplan eds. (1992) pp.1017-1024

[7] C. Hartig, X.F. Fang, H. Mecking and M. Dahms, Acta metall. mater. 408 (1992) 1883-1894

[8] D.J. Dingley, SEM Inc.IV (1981) 273-286

[9] S.A. Jones and M.J. Kaufman, Acta metall. mater. 412 (1993) 387-398 